OPEN ACCESS

Edited by:

Carlo Alviggi,

University of Naples Federico II, Italy

Reviewed by:

Matheus Roque,

Clínica Origen, Brazil

Alessandro Conforti,

University of Naples Federico II, Italy

*Correspondence:

Tatiana Chartomatsidou chartomatsidou@assistingnature.gr

Specialty section:

This article was submitted to

Reproduction,

a section of the journal

Frontiers in Endocrinology

Received: 22 March 2019

Accepted: 14 June 2019

Published: 03 July 2019

Citation:

Papanikolaou E, Chartomatsidou T, Timotheou E, Tatsi P, Katsoula E, Vlachou C, Asouchidou I, Zafeiratis O and Najdecki $R$ (2019) In Freeze-All Strategy, Cumulative Live Birth Rate (CLBR) Is Increasing According to the

Number of Blastocysts Formed in Women $<40$ Undergoing Intracytoplasmic Sperm Injection (ICSI). Front. Endocrinol. 10:427. doi: 10.3389/fendo.2019.00427

\section{In Freeze-All Strategy, Cumulative Live Birth Rate (CLBR) Is Increasing According to the Number of Blastocysts Formed in Women $<40$ Undergoing Intracytoplasmic Sperm Injection (ICSI)}

\author{
Evangelos Papanikolaou ${ }^{1,2}$, Tatiana Chartomatsidou ${ }^{1 *}$, Evangelia Timotheou ${ }^{1}$, \\ Petroula Tatsi ${ }^{1}$, Eleftheria Katsoula ${ }^{1}$, Christina Vlachou ${ }^{1}$, Irene Asouchidou ${ }^{3}$, \\ Odysseas Zafeiratis ${ }^{1}$ and Robert Najdecki ${ }^{1}$
}

${ }^{1}$ Assisting Nature, Centre of Assisted Reproduction and Genetics, Thessaloniki, Greece, ${ }^{2} 3 r d$ Department of Obstetrics and Gynecology, Aristotle University of Thessaloniki, Thessaloniki, Greece, ${ }^{3}$ Medical Department, Aristotle University of Thessaloniki, Thessaloniki, Greece

Background: Elective freezing of all embryos, followed by frozen-thawed ET cycles emerged to prevent risk of Ovarian Hyperstimulation Syndrome and to allow endometrium recovery after Controlled Ovarian Stimulation, leading to better IVF outcomes. Blastocyst Freeze-all policy can minimize the number of abnormal embryos and consequently failed ETs, but its efficacy in terms of cumulative rates has not been studied yet.

Methods: A prospective cohort observational study was carried out in Assisting Nature, Center of Assisted Reproduction and Genetics, in Thessaloniki, Greece from January 2014 until December 2017. 244 patients- normal or high responders- underwent COS with recFSH and Freeze-all policy with blastocyst culture. The included patients were 18-39 years and achieved clinical pregnancy and/or live birth or had all their vitrified blastocysts transferred in subsequent frozen-thawed cycles. Women were divided into four groups (group A: 1-2 blastocysts frozen; group B: 3-4; group C: 5-6; group D $\geq 7$ blastocysts frozen) or seven groups (group I: 1-2 blastocysts frozen, group II: 3, group III: 4, group IV: 5, group V: 6, group VI: 7; group VII: $\geq 8$ blastocysts frozen), according to the numerical range or to the absolute number of vitrified blastocysts, respectively.

Results: The main outcome of the study was the CLBR achieved by frozen-thawed ETs, according to the number of the vitrified blastocysts. Higher CLBR are expected, when at least 3 blastocysts are formed (group B: 65.2\%) and at least 2 frozen-thawed ETs are performed, reaching highest rates (88\%) by group $D$ ( $\geq 7$ vitrified blastocysts). Similarly, CLBR is significantly increasing with the absolute number of the vitrified blastocysts, ranging from $20 \%$, when $1-2$ blastocysts are vitrified (group I) to $82.4 \%$ when $\geq 8$ blastocysts are available. 
Conclusions: A higher number of vitrified blastocysts is associated with higher CLBR in women $<40$ years old- normal/high responders- following Freeze-all policy. Adopting Freeze-all strategy after blastocyst culture can contribute to improve delivery outcome after IVF, in terms of CLBR. The number of the total cryopreserved blastocysts produced might reflect the quality of the oocyte and can successfully predict the pregnancy outcome. The blastulation rate can be a robust criterion to segment or not an IVF cycle.

Keywords: freeze-all policy, blastulation rate, blastocyst, cumulative live birth rate, cumulative pregnancy rate, IVF outcome

\section{INTRODUCTION}

Although fresh embryo transfer is the golden standard in IVF and millions of couples worldwide achieved a live birth, huge progress has been recorded in various aspects of Assisted Reproduction. Especially, one main advancement is the improvement of culture conditions, allowing thus many fertilized eggs to proceed into blastocyst stage and secondly, vitrification of embryos has allowed excellent survival without any impact on their quality (1-3).

The concept of Freeze-all strategy has evolved due to two main drawbacks of multi-follicular stimulation for IVF. The first reason for the emerge of the Freeze-all strategy is safety. Ovarian Hyperstimulation Syndrome (OHSS) is considered one of the major causes of morbidity between women following Controlled Ovarian Stimulation (COS). The replacement of hCG with a GnRH agonist for oocyte maturation triggering limited the incidents of OHSS, especially among high risk patients $(4,5)$, but it is associated with lower implantation rates $(6,7)$. A GnRH antagonist protocol with a GnRH agonist trigger and Freeze-all strategy can prevent both early and late onset of OHSS, since the embryo transfer (ET) is delayed in a consecutive cycle.

The second reason is related to the endometrium environment after the COS. An increase in the number of the retrieved oocytes and consequently a higher number of available embryos is expected to contribute in a relative increase of the delivery rate $(8,9)$. However, performing fresh ET in a fresh cycle, after the COS, is correlated with lower live birth rates, if more than 20 eggs are collected $(8,10)$. Implantation is a process dependent on the embryo quality, on the endometrial receptivity and on their interaction (11). COS may enable excess number of oocytes to be retrieved and consequently fertilized, but at the same time leads to supraphysiological hormone levels during the follicular phase (12), impairing the endometrium receptivity (13-17). The segmentation of the IVF treatment in a stimulation cycle followed by Freeze-all strategy and a frozen-thawed embryo transfer in a subsequent cycle can eradicate the negative effects of COS and is associated with higher cumulative success rates (18-20).

However, it is apparent that Freeze-all policy, in order to be widely accepted and applicable, should firstly be proved more efficient than the classical policy so far, fresh first followed by thawed surplus embryos, in terms of cumulative delivery rates. Secondly, it has to be proved equally safe- if not better- in terms of health of the offsprings and with regards to the obstetrical outcome. Recent systematic reviews $(21,22)$ on babies conceived by frozen embryo transfers confirmed a lower risk of preterm delivery, small for gestational age and low birth weight. On the other hand, it has also been showed that frozen embryo transfer is associated with an increased risk of hypertensive disorders of pregnancy and large for gestational age $(21,22)$.

Regarding the offspring health it is not a matter of the current research, as we focused on the cumulative results of the stimulated ART cycles. Over the past years, much effort was put in determining the clinical outcomes of an IVF treatment regarding the number of the retrieved oocytes. Large studies supported that live birth rates are increasing with the number of retrieved oocytes, reaching a plateau after 10-15 oocytes $(8,23)$. On the other hand, a more recent study of Polyzos et al. (24) showed a continuously increase in the LBRs with the number of the oocytes, without any plateau. The study of Acharya et al. (25), based on data (2014-2015) from the American Society for Assisted Reproductive Technology Registry, showed that frozen embryo transfers benefited only patients who produced a large number of oocytes $(\geq 15)$. However, both clinical pregnancy and live birth rates in intermediate responders were higher after fresh embryo transfer than after Freeze-all and similarly the same trend was observed in poor responders, as well.

Other studies on Freeze-all policy however, suggested that improved IVF results are associated not only with cases of high responders $(12)$, but with normal responders $(17,26)$ and even with poor responders (27). Although this latter is still controversial (28-31).

The current prospective cohort study aims to identify the probability of pregnancy according to the total number of blastocysts frozen per group of patients, in cases which do not undergo fresh ET, but perform total freezing and segmentation of cycle at the blastocyst stage. Also, the study aims to provide information to patients regarding their chances to deliver a baby after a single ovarian stimulation in terms of cumulative delivery rates.

\section{MATERIALS AND METHODS}

\section{Patient Selection}

A prospective cohort observational study was performed in 254 women undergoing ICSI treatment from January 2014 until December 2017 in Assisting Nature, Center of Assisted Reproduction and Genetics, Thessaloniki, Greece. Among these women who intended to follow Freeze-all strategy, 10 patients 


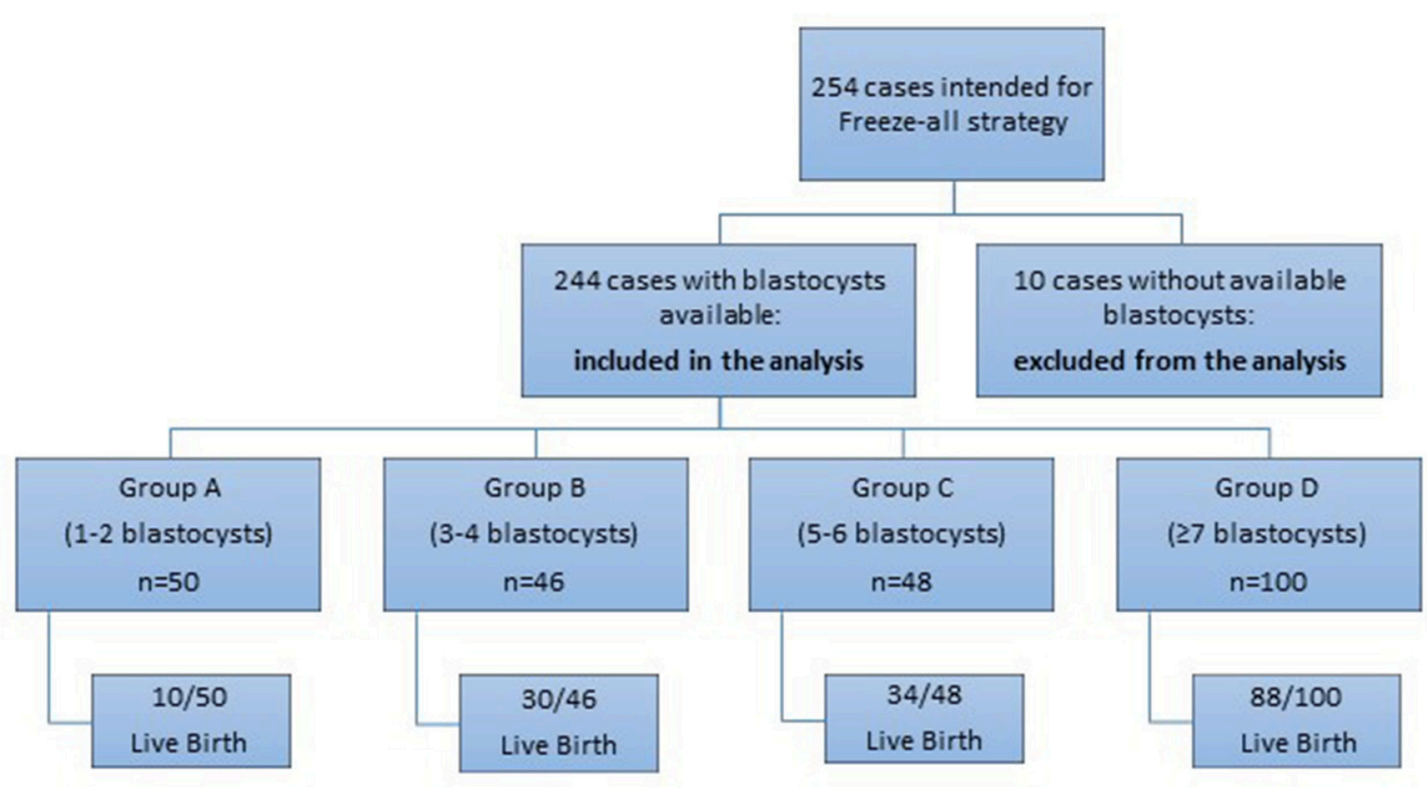

FIGURE 1 | Diagrammatic depiction of the included and excluded patients of the analysis.

had no blastocysts at the end of the embryo culture, despite that the range of the retrieved oocytes in these cases was 5-15. The rest 244 cases, which had blastocysts available for vitrification were included in the analysis (Figure 1). All the studied cases concerned couples with a female age range of 18-39 years and with fresh ejaculated sperm. The recruitment of the patients for the Freeze-all strategy was performed at the day of egg retrieval and only if the retrieved oocytes of a patient were $>4$. Exclusion criteria were: donor oocyte programs, PGS/PGD cycles, women with $\leq 4$ oocytes per retrieval and cases with sperm retrieved by TESE. Written informed consent was signed and obtained by all the participants of the study. The study was registered in clinicaltrials.gov under the registration number NCT03463278.

\section{Ovarian Stimulation}

All patients were treated with GnRH antagonist protocol, as part of the COS of IVF treatment. Gonadotrophins (Gonal$\mathrm{F}^{\circledR}$; Serono, Hellas) were administered from day- 2 of the cycle, if the hormonal levels were basal. The initial dose of the gonadotrophins was predefined at 150-300 IU for all patients and remained fixed for 5 days. After this period, the dose could be adjusted to each patient, according to the follicular growth and the estradiol levels.

Co- treatment with GnRH antagonist started on Day 6 of the stimulation with $0.25 \mathrm{mg}$ cetrorelix (Merck-Serono) or $0.25 \mathrm{mg}$ ganirelix (MSD).

The final oocyte maturation was induced when at least 3 follicles of $18 \mathrm{~mm}$ were present during ultrasound examination. Oocyte retrieval was performed $36 \mathrm{~h}$ after the administration of the triggering regimen. When $<14$ follicles were present, rec-HCG (Ovitrelle ${ }^{\circledR}$; Merck-Serono) was administered.
Alternatively, if $\geq 14$ follicles were present, then agonist triggering was chosen with $0.3 \mathrm{mg}$ Triptorelin (Arvekap ${ }^{\circledR}$, IPSEN).

\section{Embryological Data}

The incubation conditions of the embryo culture were set at $6 \%$ $\mathrm{CO}_{2}, 5 \% \mathrm{O}_{2}$ and $37^{\circ} \mathrm{C}$. The culture of the embryos was supported by the sequential media provided by Origio ${ }^{\circledR}$ (Cleavage medium and Blastocyst medium). Zygotes were scored on day 1 after ICSI, based on the presence of the 2 pronuclei (2PN) and their morphology (32). Embryo quality was assessed on the morning of day 3 according to the number of the cells, the equality of the blastomere size and the fragmentation rate. Embryos were also evaluated on day 5 according to the embryo scoring system of Gardner and Schoolcraft (33), regarding the formation and the morphology of the blastocysts. The quality of the blastocysts was characterized as top (grade 3-5AA, 3-5AB), good (3-5BA, 3-5BB) or fair (1-2AA, 1-2AB, 1-2BA, 1-2BB). The proportion of each embryo quality in every studied group is mentioned in Table $\mathbf{1}$.

Sperm preparation and ICSI procedure were carried out as described by Van Landuyt et al. (34).

The cryopreservation of the blastocysts was conducted on the fifth or the sixth day of the culture. In the studied cases, there were no incidents of day 6 blastocysts to be included in the final vitrified blastocyst cohort. Vitrification and thawing of the cryopreserved blastocysts were performed with the Kitazato ${ }^{\circledR}$ Vitrification Kit and Kitazato ${ }^{\circledR}$ Warming Kit, respectively. The vitrification carrier used was the Cryotec straw by Cryotech ${ }^{\circledR}$ Japan.

\section{Endometrial Preparation for FET}

None of the included cases were submitted to fresh ET. The vast majority of patients underwent estrogen replacement 
TABLE 1 | The proportion of top, good, and fair quality blastocysts vitrified in the overall blastocyst number $(N)$ per each group.

\begin{tabular}{lcccc}
\hline Blastocyst quality & $\begin{array}{c}\text { Group A } \\
(\mathbf{1 - 2} \text { Blastos frozen) } \\
\mathbf{N = 9 2}\end{array}$ & $\begin{array}{c}\text { Group B } \\
\text { (3-4 Blastos frozen) } \\
\mathbf{N = 1 6 4}\end{array}$ & $\begin{array}{c}\text { Group C } \\
\text { (5-6 Blastos frozen) } \\
\mathbf{N = 2 6 2}\end{array}$ & $\begin{array}{c}\text { Group D } \\
\mathbf{2} \text { B Blastos frozen) } \\
\mathbf{N = 9 5 2}\end{array}$ \\
\hline Top & $63 \%(58 / 92)$ & $67.1 \%(110 / 164)$ & $80.9 \%(212 / 262)$ & $83.4 \%(794 / 952)$ \\
Good & $17.4 \%(16 / 92)$ & $19.5 \%(32 / 164)$ & $12.2 \%(32 / 262)$ & $12.6 \%(120 / 952)$ \\
Fair & $19.6 \%(18 / 92)$ & $13.4 \%(22 / 164)$ & $6.9 \%(18 / 262)$ & $4 \%(38 / 952)$
\end{tabular}

preparation of endometrium and only $1 \%$ underwent natural cycle synchronization for transfer. Women who followed estrogen replacement preparation of the endometrium started hormonal monitoring on day- 2 of the cycle and if estradiol was $<80 \mathrm{pg} / \mathrm{ml}$, progesterone was $<1.5 \mathrm{ng} / \mathrm{ml}$ and no cyst was present on the ultrasound scan then the patient could start the treatment. On this day women started $17 \beta$ - estradiol (Divina ${ }^{\circledR}$; Orion Corporation or Cyclacur ${ }^{\circledR}$; Bayer Hellas) with $2 \mathrm{mg}$ once in the night of day- 2 and then $4 \mathrm{mg}$ from day- 3 to day- 5 and increasing to $6 \mathrm{mg}$ from day- 6 to day- 8 and then increasing again to $8 \mathrm{mg}$ from day- 9 to day- 11 onwards. On day-11 ultrasound scan and hormone level (E2, PG) test was performed. If the endometrial thickness was $>7 \mathrm{~mm}$, the estrogen level $>150 \mathrm{pg} / \mathrm{ml}$ and the progesterone level $<1.5 \mathrm{ng} / \mathrm{ml}$ (35), then the progesterone supplementation could be started upon the planning of the embryology lab. The estrogen dose was decreased to 6 and $600 \mathrm{mg}$ progesterone in 3 doses of $200 \mathrm{mg}$ for 6 days were administered in order to transfer blastocysts.

When the embryo replacement was conducted in a natural cycle, the monitoring started on the first day of the cycle. An ultrasound scan was conducted on day 3-4, then on day-9 and repeated on day 11-12 along with hormone levels test. The day of the transfer was decided upon definition of ovulation day, based upon monitoring the rise of $\mathrm{LH}$ above $14 \mathrm{mIU} / \mathrm{ml}$ or progesterone rise above $1.5 \mathrm{ng} / \mathrm{ml}$.

The pregnancy test was performed 14 days after the progesterone supplementation initiation. In case of a negative test, the treatment was discontinued. Alternatively, in case of pregnancy, the treatment continued until 10 weeks and then gradually the medications discontinued.

\section{Embryo Transfer Policy}

The policy of the embryo transfer of our clinic was in accordance to the Greek law. Elective double embryo transfer was offered and allowed by law. Nevertheless, if the patient's age was $<32$ years old, or upon the couple's request, then single embryo transfer was chosen, although the law still was allowing double ET.

\section{Cumulative Rates Per Individual}

With the evolution of the Freeze-all strategy, a new index for evaluating the success of an ART cycle is needed. In this study, a modified index is used, based on the proposed COMFFETI (Combined Fresh and Frozen Embryo Transfers per Individual) by Papanikolaou et al. (36), which is a binomial variable (yes or no) that reflects the achievement of a pregnancy by individual (couple), after each stimulated cycle. COMFFETI index originally includes results by both fresh (when performed) and thawed ET (when performed), obtained by a single ovarian stimulation cycle, assessing the success of both fresh and frozen cycles performed by the same woman.

The cases included in the present study did not perform fresh ET, but followed Freeze-all strategy at the blastocysts stage and subsequent frozen- thawed ETs. As a result, the cumulative results of the studied cases derive only from FET cycles. The cumulative result of "clinical pregnancy" was estimated if clinical pregnancy was achieved in any of the frozen-thawed treatments, which resulted after a single ovarian stimulation. Similarly, if any of the FET cycles of a woman resulted in a delivery of a baby, then this particular couple is considered as a case which achieved "live birth." The total number of the studied cases was 244 . All patients performed up to 3 frozen-thawed ETs, except from 6 cases which performed up to 4 .

For the first part of the study, patients were divided in 4 groups, according to the number of the vitrified blastocysts at the end of the stimulation cycle. Group A consists of women with 1-2 blastocysts frozen; group B includes women with 3-4 blastocysts; group C consists of women with 5-6 blastocysts frozen and group $\mathrm{D}$ refers to women with 7 or more blastocysts frozen.

For reasons of further analysis, these patients were also divided in 7 groups, according to the absolute number of vitrified blastocysts after the stimulation cycle; group I: 1-2 blastocysts frozen, group II: 3 blastocysts frozen, group III: 4 blastocysts frozen, group IV: 5 blastocysts frozen, group V: 6 blastocysts frozen, group VI: 7 blastocysts frozen and group VII: more than 7 blastocysts frozen.

The success rates of the ART cycles were estimated as Cumulative Rates. Cumulative Rates are estimated as the probability of achieving a clinical pregnancy/ live birth after one single stimulation cycle by transferring the available embryos in subsequent frozen- thawed ETs.

The Cumulative Clinical Pregnancy Rate (CCPR) and Cumulative Live Birth Rate (CLBR) were estimated separately for each group of patients. The included cases either achieved a clinical pregnancy/ live birth or had all their vitrified/ thawed blastocysts transferred after completing 3 cycles of frozen-thawed ETs (except from 6 cases with up to 4 FET).

\section{Statistical Analysis}

The data from the statistical analysis are presented as mean \pm standard deviation or percentage. The statistical evaluation was performed with SPSS software (SPSS, Chicago, IL, USA). Descriptive statistics were performed for each variable and the quantitative results are performed as the mean $( \pm S D)$. 
Means were compared by using ANOVA and t-test, while proportions between the groups were compared using chi-square test. Differences were considered significant when $p<0.05$.

\section{Ethical Approval}

This prospective cohort observational study was approved by the Institutional Review Board of Assisting Nature, Center of Assisted Reproduction and Genetics, Thessaloniki, Greece.

\section{RESULTS}

\section{Patients Characteristics}

Overall, the analysis included 244 cases divided in different groups, according to the number of the available vitrified

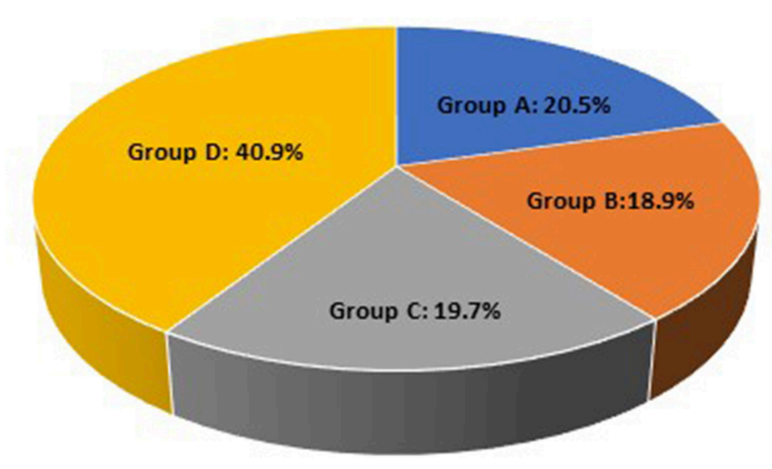

- Group A (1-2 Blastos Frozen) - Group B (3-4 Blastos Frozen)

" Group C (5-6 Blastos Frozen) " Group D ( $\geq 7$ Blastos Frozen)

FIGURE 2 | The frequency of each study group; Group A (1-2 blastocysts frozen), Group B (3-4 blastocysts frozen), Group C (5-6 blastocysts frozen) and Group D ( $\geq 7$ blastocysts frozen) on the total case number. blastocysts. Overall, the analysis included 244 cases. For the first part of the study, patients were divided in 4 groups, according to the number of the vitrified blastocysts at the end of the stimulation cycle: Group A (1-2 blastocysts frozen) included 50 patients (20.5\%), Group B (3-4 blastocysts frozen) included 46 cases (18.9\%), Group C (5-6 blastocysts frozen) comprised of 48 cases $(19.7 \%)$ and the last Group D ( $\geq 7$ blastocysts frozen) included 100 cases $(40.9 \%)$ of the total population studied (Figure 2).

No complications, such as events of OHSS, were noticed after the COS of these patients. As a result, 36.1\% (88/244) of the patients received HCG triggering and 63.9\% (156/244) agonist triggering.

A rate of $96 \%$ of the cases underwent elective double embryo transfer, while $2 \%$ had elective single ET (elective SET) and $2 \%$ SET. In the latter case, this $2 \%$ of the women followed SET, because no more blastocysts were available.

The mean values of age, COCs (Cumulus- Oocyte Complex) and metaphase 2 (MII) oocytes are shown on Table 2. Although the mean age of the women ranged from 31.9, to 34.5 , and the differences among groups did not exceed the 3 years, there was statistical difference among groups.

The mean number of the COCs and the MII oocytes after the oocyte retrieval of the studied cases are also significantly different between the groups $(p<0.05)$.

As it would obviously be anticipated, the number of the COCs was significantly correlated with the number of the MII oocytes and the number of the blastocysts available for vitrification, at a significance level of 0.05 .

A logistic regression analysis for the four groups was carried out with dependent variable being the achievement of live birth or not and independent variables were the age, the mean number of COCs and the blastulation rate. The analysis showed that the only variable remaining significant was the number of the COCs $(p<0.05)$.

TABLE 2 | The mean values and Standard Deviation (SD) of age, basal FSH, reason of infertility, number of COCs, number of MIl oocytes, fertilization and blastulation rates, and post-thawing survival rate of the blastocyss of the groups, characterized by the statistical significance: Group A (1-2 blastocysts frozen), Group B (2-3 blastocysts frozen), Group C (5-6 blastocysts frozen) and Group D ( $\geq 7$ blastocysts frozen).

\begin{tabular}{|c|c|c|c|c|c|c|}
\hline & & $\begin{array}{c}\text { Group A } \\
\text { (1-2 Blastos Frozen) } \\
(n=50)\end{array}$ & $\begin{array}{c}\text { Group B } \\
\text { (3-4 Blastos Frozen) } \\
(n=46)\end{array}$ & $\begin{array}{c}\text { Group C } \\
\text { (5-6 Blastos Frozen) } \\
(n=48)\end{array}$ & $\begin{array}{c}\text { Group D } \\
(\geq 7 \text { Blastos Frozen) } \\
(n=100)\end{array}$ & $\begin{array}{c}\text { s.s }{ }^{b} \\
\text { (p-value) }\end{array}$ \\
\hline \multicolumn{2}{|c|}{ Age $^{\mathrm{a}}$ mean $( \pm S D)$} & $31.84( \pm 5.21)$ & $34.48( \pm 3.42)$ & $33.96( \pm 3.69)$ & $32.12( \pm 3.66)$ & $P<0.05$ \\
\hline \multicolumn{2}{|c|}{ Basal FSH mean (mIU/ml) } & 7.1 & 6.9 & 6.7 & 6.1 & n.s. \\
\hline \multirow[t]{3}{*}{ Infertility reason } & Male factor & $40 \%$ & $42 \%$ & $44 \%$ & $45 \%$ & n.s. \\
\hline & Female factor & $39 \%$ & $36 \%$ & $36 \%$ & $32 \%$ & n.s. \\
\hline & Both & $21 \%$ & $22 \%$ & $20 \%$ & $23 \%$ & n.s. \\
\hline \multicolumn{2}{|c|}{ COCs mean (range) } & $9.80(5-24)$ & $11.61(5-21)$ & $11.96(6-21)$ & $18.66(7-58)$ & $P<0.05$ \\
\hline \multicolumn{2}{|l|}{ MII mean ( $\pm S D)$} & $6.56( \pm 3.56)$ & $8.52( \pm 2.84)$ & $9.52( \pm 2.91)$ & $14.66( \pm 5.18)$ & $P<0.05$ \\
\hline \multicolumn{2}{|c|}{ Fertilization rate mean $( \pm S D)$} & $81.74 \%$ (15.66\%) & $79.57 \%( \pm 16.04 \%)$ & $85.46 \%( \pm 10.35 \%)$ & $86.94 \%( \pm 11.39 \%)$ & n.s \\
\hline \multicolumn{2}{|c|}{ Blastulation rate mean $( \pm S D)$} & $48.22 \%( \pm 26.1 \%)$ & $59.24 \%( \pm 18.67 \%)$ & $72.95 \%( \pm 17.95)$ & $79.55 \%( \pm 20.58)$ & $P<0.05$ \\
\hline \multicolumn{2}{|c|}{ Post-Thawing Survival rate mean $( \pm S D)$} & $100 \%( \pm 0.1 \%)$ & $100 \%( \pm 0.1 \%)$ & $100 \%( \pm 0.1 \%)$ & $100 \%( \pm 0.1 \%)$ & n.s \\
\hline
\end{tabular}

${ }^{a}$ Age is expressed in years.

b Statistical significance. 


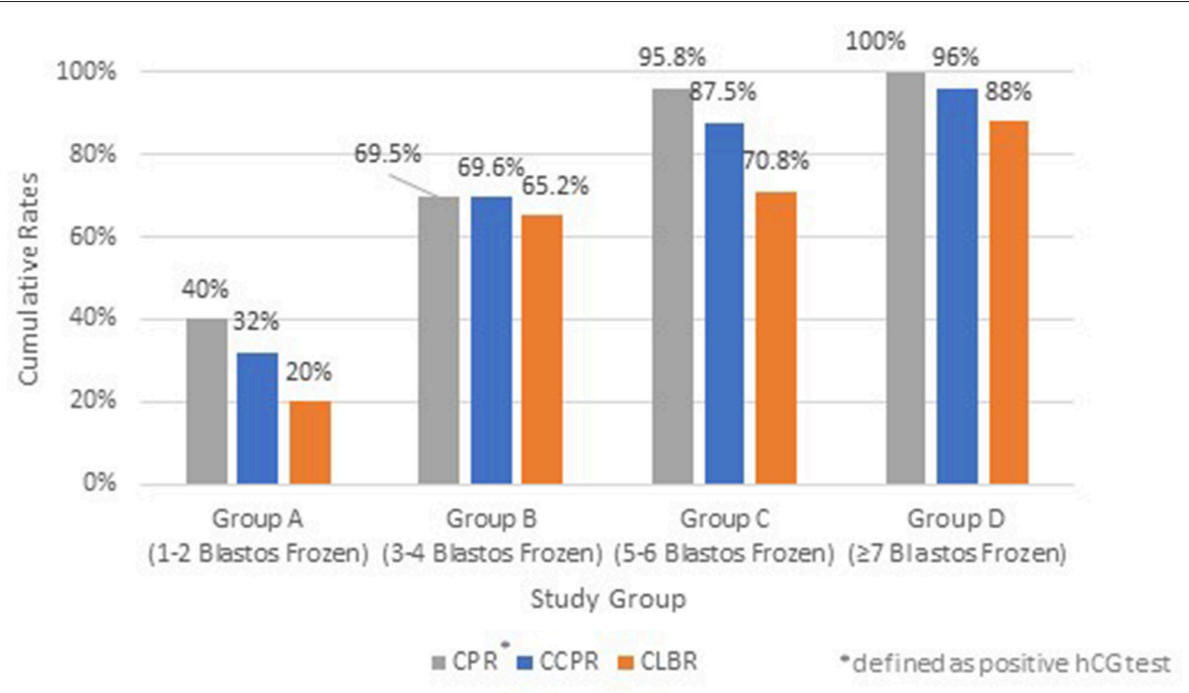

FIGURE 3 | Cumulative Pregnancy Rate (CPR) ${ }^{\star}$, Cumulative Clinical Pregnancy Rate (CCPR) and Cumulative Live Birth Rate (CLBR) of each study group; Group A (1-2 blastocysts frozen), Group B (3-4 blastocysts frozen), Group C (5-6 blastocysts frozen), and Group D ( $\geq 7$ blastocysts frozen). *Defined as positive hCG test.

Initially, 254 women were intended to follow Freeze-all strategy. Ten out of these women did not have any available blastocyst at the end of the embryo culture (even until the sixth day). All 10 women had 5-15 oocyte yield after the retrieval. Maturation and fertilization rate in 7 out of 10 cases were comparable with the rest of the sample. One of these 7 women got pregnant in a subsequent IVF treatment, after an additional COS cycle; 4 continued the treatment unsuccessfully so far; and the rest two accepted oocyte donation achieving pregnancy and live birth.

Maturation problem appeared in the rest 3 out of the 10 patients (16.7-53.3\%). One of these women continued the trials, despite the fact that she had already undergone two IVF therapies, in other clinics, without ET. The two other cases facing maturation problem have not gotten pregnant and refused oocyte donation.

\section{Fertilization and Blastulation Rate}

The fertilization rate was calculated by the number of the $2 \mathrm{PN}$ embryos divided by the total number of the MII oocytes, in each case. For the calculation of the blastulation rate, the dominator was the total number of the $2 \mathrm{PN}$ embryos. The mean fertilization and blastulation rates of each group are presented on Table 2. The statistical analysis showed no difference in the mean value of the fertilization rate between the groups, while there is a significant difference of the mean rates between the four groups $(p<0.05)$, regarding the blastulation rate.

\section{Cumulative Clinical Pregnancy Rate (CCPR) and Cumulative Live Birth Rate (CLBR)}

The impact of the different number of available blastocysts for Freeze-all strategy after an ICSI cycle on CCPR and CLBR are summarized on Figure 3. Patients with 1-2 blastocysts vitrified achieved low CCPR of 32\%, whereas the other groups showed 69.6\% (Group B), 87.5\% (Group C), and 96\% (Group D), respectively, which is proven statistically significant $(p<0.05)$.

The CLBR for each group of patients are also depicted in Figure 3. The CLBR rose from 20\%, when only 2 blastocysts were cryopreserved, up to $88 \%$ in group D with more than 7 blastocysts available $(p<0.05)$, taken into account that in this group some couples underwent second or third or even fourth embryotransfer in order to achieve such high CLBRs.

There were no incidents of triplets in the studied cases. The rate of twin deliveries was $33.3 \%(54 / 162)$ and the rate of the singleton deliveries was $66.7 \%(108 / 162)$ in the women of all groups (Table 3 ).

\section{Contribution of Each FET Cycle to the Cumulative Delivery Rate}

The cumulative increase in the delivery rates after each FET was calculated for each group of patients. The results of the cumulative effect of any additional FET cycle are presented on Table 4 and Figure 4.

Group A had a delivery rate of $20 \%$ after the first frozenthawed cycle, which was also the total cumulative rate, as the majority of the cases had embryos available for only one FET. In this group only 4 out of 50 patients performed single embryo transfer and therefore had a second FET. However, none of these FET treatments resulted in a delivery (these patients had decided to vitrify their embryos one by one).

Group B achieved 56.5\% delivery rate after the first FET and the rate was increased by a $4.3 \%$ after the second FET cycle and additionally by $4.4 \%$ after the third one, reaching thus a CLBR of $65.2 \%$. This increase in the delivery rate after the three sequential FET cycles is not considered significant $(p>0.05)$. 
TABLE 3 | The rate of singleton and twin deliveries in the live birth cases $(\mathrm{N})$ in each group and in the overall live birth cases.

\begin{tabular}{|c|c|c|c|c|c|}
\hline & $\begin{array}{c}\text { Group A } \\
\text { (1-2 Blastos frozen) } \\
N=10\end{array}$ & $\begin{array}{c}\text { Group B } \\
\text { (3-4 Blastos frozen) } \\
N=30\end{array}$ & $\begin{array}{c}\text { Group C } \\
\text { (5-6 Blastos frozen) } \\
N=34\end{array}$ & $\begin{array}{c}\text { Group D } \\
\text { ( } \geq 7 \text { Blastos frozen) } \\
N=88\end{array}$ & $\begin{array}{c}\text { Overall } \\
N=162\end{array}$ \\
\hline Twin delivery rate & $\begin{array}{l}20 \% \\
(2 / 10)\end{array}$ & $\begin{array}{l}33.3 \% \\
(10 / 30)\end{array}$ & $\begin{array}{c}41 \% \\
(14 / 34)\end{array}$ & $\begin{array}{l}31.8 \% \\
(28 / 88)\end{array}$ & $\begin{array}{c}33.3 \% \\
(54 / 162)\end{array}$ \\
\hline
\end{tabular}

TABLE 4 | Live Birth Rate (LBR) after each FET cycle and Cumulative Live Birth Rate (CLBR) after each FET cycle of the studied groups according to the number of vitrified blastocysts.

\begin{tabular}{|c|c|c|c|c|c|c|c|c|c|}
\hline & $\begin{array}{l}\text { LBR after } \\
\text { FET } 1\end{array}$ & CLBR 1 & $\begin{array}{l}\text { LBR after } \\
\text { FET } 2\end{array}$ & CLBR 2 & $\begin{array}{l}\text { LBR after } \\
\text { FET } 3\end{array}$ & CLBR 3 & $\begin{array}{l}\text { LBR after } \\
\text { FET } 4\end{array}$ & CLBR 4 & Total CLBR \\
\hline $\begin{array}{l}\text { Group A } \\
(1-2 \text { Blastos Frozen) } \\
(n=50)\end{array}$ & $\begin{array}{c}20 \% \\
(10 / 50)\end{array}$ & $\begin{array}{c}\mathbf{2 0} \% \\
(10 / 50)\end{array}$ & $\begin{array}{l}0 \% \\
(0 / 4)\end{array}$ & $\begin{array}{c}20 \% \\
(10 / 50)\end{array}$ & N/A & N/A & $\mathrm{N} / \mathrm{A}$ & N/A & $\begin{array}{c}20 \% \\
(10 / 50)\end{array}$ \\
\hline $\begin{array}{l}\text { Group B } \\
\text { (3-4 Blastos Frozen) } \\
(n=46)\end{array}$ & $\begin{array}{l}56.5 \% \\
(26 / 46)\end{array}$ & $\begin{array}{l}\mathbf{5 6 . 5 \%} \\
(26 / 46)\end{array}$ & $\begin{array}{l}14 \% \\
(2 / 14)\end{array}$ & $\begin{array}{l}60.8 \% \\
(28 / 46)\end{array}$ & $\begin{array}{c}100 \% \\
(2 / 2)\end{array}$ & $\begin{array}{l}65.2 \% \\
(30 / 46)\end{array}$ & $\mathrm{N} / \mathrm{A}$ & $\mathrm{N} / \mathrm{A}$ & $\begin{array}{l}65.2 \% \\
(30 / 46)\end{array}$ \\
\hline $\begin{array}{l}\text { Group D } \\
(\geq 7 \text { Blastos Frozen) } \\
(n=100)\end{array}$ & $\begin{array}{c}62 \% \\
(62 / 100)\end{array}$ & $\begin{array}{c}\mathbf{6 2} \% \\
(62 / 100)\end{array}$ & $\begin{array}{l}64.7 \% \\
(22 / 34)\end{array}$ & $\begin{array}{c}84 \% \\
(84 / 100)\end{array}$ & $\begin{array}{l}33 \% \\
(4 / 12)\end{array}$ & $\begin{array}{c}88 \% \\
(88 / 100)\end{array}$ & $\begin{array}{l}0 \% \\
(0 / 6)\end{array}$ & $\begin{array}{c}88 \% \\
(88 / 100)\end{array}$ & $\begin{array}{c}88 \% \\
(88 / 100)\end{array}$ \\
\hline
\end{tabular}

N/A, Not Applicable. The bold values represent the CLBR after each FET cycle.

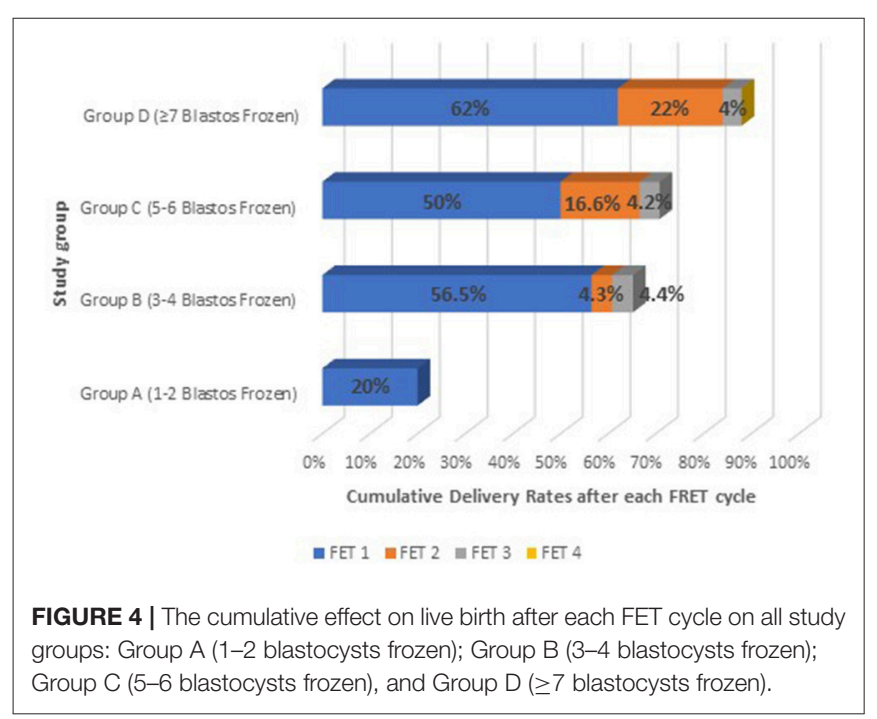

Group C showed an even higher increase in the Live Birth Rate after the second and the third FET. The delivery rate was significantly increased by $16.6 \%$ after the second FET $(p<0.05)$ and by only $4.2 \%$ after the third FET, reaching a total CLBR of $70.8 \%$.

Group D was the only group which included patients with number of embryos available also for a fourth FET. Patients in that group had $62 \%$ delivery rate after the first FET, which was significantly increased by $22 \%(p<0.05)$ after the second FET and by $4 \%$ after the third. The total CLBR for these patients was $88 \%$. Despite that in this group there were 6 patients who had even a fourth frozen- thawed ET and although all six of them achieved a biochemical pregnancy in one out of the four transfers, finally none of them managed to deliver.

\section{Cumulative Live Birth Rates According to the Absolute Number of Vitrified Blastocysts}

For reasons of further analysis, patients were also divided in 7 groups, according to the absolute number of vitrified blastocysts after the stimulation cycle; group I: 1-2 blastocysts frozen ( $n=$ $50)$, group II: 3 blastocysts frozen $(n=20)$, group III: 4 blastocysts frozen $(n=26)$, group IV: 5 blastocysts frozen $(n=26)$, group V: 6 blastocysts frozen $(n=22)$, group VI: 7 blastocysts frozen $(n=$ 32 ) and group VII: more than 7 blastocysts frozen $(n=68)$.

The Cumulative Live Birth Rates were further analyzed, according to the absolute number of vitrified blastocysts (Figure 5). Group I (1-2 blastocyst frozen) and Group II (3 blastocysts frozen) showed significantly different CLBRs of $20 \%$ $(n=50)$ and $50 \%(n=20)$, respectively. Group III (4 blastocysts frozen) had higher rates of $76.9 \%(n=26)$ and from this point and after the respective rates for the other groups with $5(n=$ $26)$ and $6(n=22)$ blastocysts frozen did not differ statistically, as they were similar to that of Group III (61.5 and $81.8 \%$, 


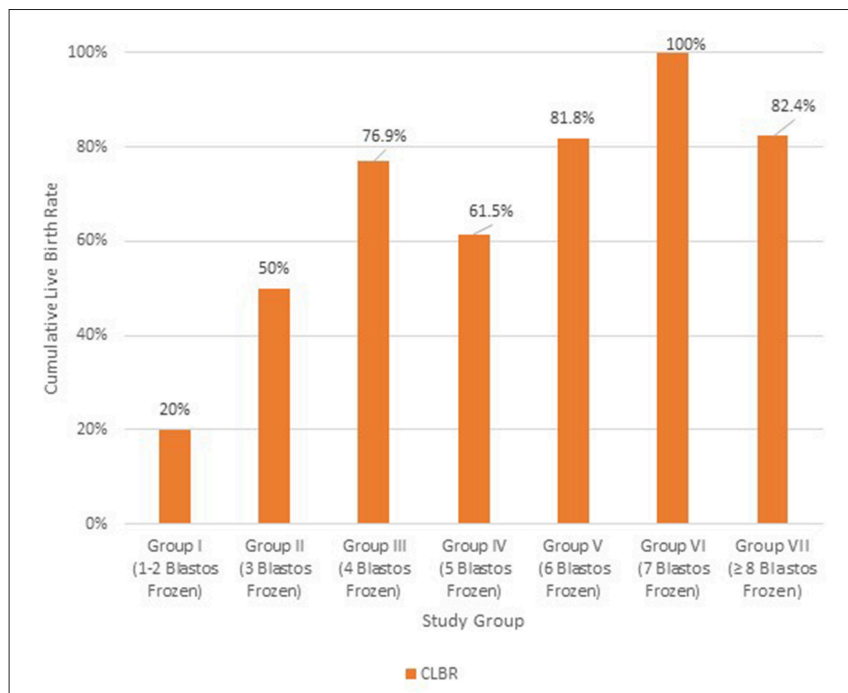

FIGURE 5 | Cumulative Live Birth Rate (CLBR) in each group of patients according to the absolute number of vitrified blastocysts; Group I: 1-2 blastocysts frozen ( $n=50)$; Group II: 3 blastocysts frozen $(n=20)$; Group III: 4 blastocysts frozen ( $n=26)$; Group IV: 5 blastocysts frozen $(n=26)$; Group V: 6 blastocysts frozen ( $n=22)$; Group VI: 7 blastocysts frozen $(n=32)$; Group VII: $\geq 8$ blastocysts frozen $(n=68)$.

respectively). A significant increase in the CLBR was shown again when 7 blastocysts were available $(n=32)$. At this point the CLBR was $100 \%$ and the rate was significantly decreasing when more than $7(n=68)$ blastocysts were available $(82.4 \%)$. In the latter case, the rates were similar with the CLBR of the group with 6 vitrified blastocysts (group V).

\section{DISCUSSION}

Embryo transfer in a fresh cycle, after the stimulation, has been for years the golden standard in IVF, despite the definite risk for OHSS, the definite negative effect on endometrial receptivity and despite the plausible negative impact on the achievement of a pregnancy. Looking from a cumulative delivery point of view, a fresh ET might sacrifice a euploid embryo in an unproper matured endometrium, whereas, a first time frozen embryo transfer may offer the best quality endometrium environment, increasing thus the cumulative probability of having a baby. The reason for not practicing all these years the segmentation of cycle in two parts (one fresh including stimulation, egg retrieval, fertilization and embryo culture and a second one including one or more thawed embryo transfers), was the modest survival rate after slow freezing, the even lower pregnancy rates after replacing thawed slow frozen embryos (37) and the inconclusive literature on the outcome of offsprings of frozen embryos $(38,39)$.

The emerging policy of Freeze-all is becoming more widely accepted as it is considered a safer treatment, especially for OHSS high risk patients (40) and under the wider acceptance and credibility that vitrification technique has gained $(1,18,26,37$, 41-43), due to excellent post- thawing survival of the embryos and of the better synchrony of the endometrium cavity (10). Moreover, it has been suggested that Freeze-all strategy can lead to improved IVF results, when used individualized, according to each patient case (44).

The present study aimed to evaluate the efficacy of Freeze-all strategy in terms of Cumulative Live Birth Rates, according to the number of the blastocysts which were available for vitrification. For reasons of objectivity, the study did not include cases of poor responders, but only normal and high responders. Even patients of Group A, who had 1-2 blastocysts available for vitrification, were categorized as normal responders (5-24 oocytes retrieved). It should be noted that no embryo loss was observed in the studied cases, indicating the excellent efficacy of vitrification/ thawing process, at least in our hands.

It was expected that younger women especially with male infertility factor would form the group with high number of blastocysts. Nevertheless, such scenario was not the case in our sample. On the contrary, women of group A -having the lowest number of available blastocysts (1-2)- were the patients with the youngest mean age, which was surprisingly similar to the mean age of women of group D (more than 7 blastocysts available). The only statistically significant difference was observed between women of group A and group B., apparently by a matter of randomness.

In contrast to previous studies $(8,9,23-25,37,45-48)$ which were based on the number of retrieved COCs to evaluate the success of an IVF treatment, the present research was designed upon the number of transferable blastocysts. Taking the decision -whether to transfer or segment- on the fifth day of embryo culture is more robust as the information provided about couple's implantation potential on that particular cycle is closer to reality. It is interesting that, in our analysis the mean number of the retrieved COCs differs significantly only in the case of group D ( $\geq 7$ blastocysts) compared to all the other groups. Indeed, the number of the retrieved oocytes is similar between groups A, B and $\mathrm{C}$. Although there is no significant difference in the oocyte number among these three groups, it is essential to focus on the clinical results that eventually rise form the different number of the formed blastocysts.

The results of the analysis have shown a significant increase in the Cumulative Live Birth Rates with the number of vitrified blastocysts. Indeed, patients in Group B achieved more than a triple rate of cumulative live birth compared to patients of Group A $(20$ vs. $65.2 \%, p<0.05)$ and statistically similar results are obtained for Group C (70.8\%). Unsurprisingly, the highest CLBR, as expected, was achieved by couples of Group D (88\%), which had more than 7 blastocysts vitrified, but also were mostly high responders (Table 4 ).

The above results suggest that higher CLBRs can be expected when at least 3 blastocysts are formed. It appears that a total of three blastocysts is the threshold for achieving a CLBR of at least 50\% (Figure 5). Obviously, live birth rates above 80\% were noticed for patients with up to 6 vitrified blastocysts, implicating that at least 2 FET cycles are vital for higher clinical results. Indeed, for each extra 2 blastocysts available above two, the cumulative delivery rate was increasing. The extremely high cumulative live birth rate of $88 \%$ (Group D) 
when the couple segments the cycle and performs repetitive thawed embryo transfers, indicates first of all, the relative reproductive health of these couples, but also the potential of the Freeze-all strategy.

Patients of Group D ( $\geq 7$ blastocysts frozen) achieved a live birth rate of $62 \%$ after the first FET and cumulatively after the third FET reached a level of $88 \%$ of live births. Although all women in this particular group had a positive hCG test, of those 12 who did not manage to deliver, four (33.3\%) had a biochemical pregnancy and eight $(66.6 \%)$ had a clinical pregnancy which ended as an abortion in $<12$ weeks. The above results indicate that high availability of transferable blastocysts might be related to better quality as well, and at the end those who did not give birth might be cases with other co-existing infertility factors related mainly to the mother.

Another interesting finding of our study is that although a significant increase exists in live birth rates between the first and the second FET, however, this effect is decreasing when a third or fourth FET cycle is required. This modest increase after a third embryo transfer (Figure 4) could possibly reflect the diminishing quality of the remaining embryos, as the morphologically best and thus most implantable blastocysts are usually preferred to be transferred first. Indeed, a fourth FET for 6 patients of Group D did not led to delivery, at any case.

On the contrary, the cumulative rates of Group A correspond to solely one FET cycle, limiting thus the possibility of a cumulative increase due to consequent frozen-thawed ETs. In this group, there was an exception of 4 patients who completed two single embryo transfers, as they had chosen to have their (two) embryos vitrified one by one. However, no clinical pregnancy was achieved in those cases. We have to underline that the fertilization rate of Group A was not statistically different, but the blastulation rate was significantly lower, comparing to the groups with higher number of blastocysts (Group C and Group D).

The conflicting results of previous studies do not support profoundly the prediction of the outcome of an IVF treatment based on the concept of the ovarian response of a woman (24, $49,50)$. The present analysis proposes a new concept, which in contrary to previous research, is based on the blastulation rate, instead. The study of the number of the oocytes may reflect the ovarian response of a woman, but does not take into consideration other factors implicating in the embryo development. The results show that a more solid indicator on the potential efficacy of an ART cycle appears to be the blastulation rate, which reflects not only the impact of the pre-fertilization aspects (oocyte quality), but also of all the post- fertilization factors (sperm genetic quality, embryo genome activation, culture conditions, etc.). The combination of many parameters contributes to the formation of the blastocysts, which may have implantation potential. Thus, the ability of the zygotes to reach the blastocyst stage is the most promising indicator, regarding the clinical outcomes after a single oocyte retrieval.

Moreover, although Rubio et al. suggested that the percentage of the euploid embryos seems to decrease, when more oocytes are retrieved (51), others (52) support that a higher number of euploid embryos is observed when higher number of oocytes is retrieved. On the other hand, it has also been suggested that although the rate of euploid embryos remains stable, regardless the number of the available embryos $(53,54)$, every additional embryo significantly increases the chances of a woman to have at least one normal embryo (53). Consequently, the above literature agrees with our results, showing higher cumulative rates when more transferable blastocysts are available.

Adopting a Freeze-all strategy- besides the possible advantageous impact on the clinical results- is essential in preventing OHSS (40), especially in women with high oocyte yield, making the IVF treatment a safe procedure. Nevertheless, this strategy has not been proved beneficial for patients with less than two blastocysts available. A fresh embryo transfer in these cases might guarantee the same or even better results than the Freeze-all policy.

It is obvious, however, that the universal use of Freezeall policy at the blastocyst stage, requires standardized and continuously controlled lab conditions, that may allow the best embryos to reach the ultimate in vitro developmental point. Moreover, an expertised lab personnel is essential to ensure successful vitrification and thawing procedures, that can guarantee embryo survival in a rate of more than $95 \%$. Therefore, adopting strict lab quality control, any possible external factors that could affect the culture and the quality of the forming embryos, could be eliminated.

Another interesting finding was the high twin rate (>30\%) in the last three groups (Table 3 ). This result indicates that since the cumulative delivery rate is really high, a viable option could be to implement elective single embryo transfer when more than 4 blastocysts are available, even if the time to delivery would prolongated with such policy. Since the cumulative delivery rate should remain equal, the twin rate would decrease dramatically to levels $<1.5 \%$ and thus decreasing complications related with plausible preterm labors.

Currently, an indiscriminate submission of all IVF/ICSI patients to Freeze-all strategy and subsequent FET cycles is not supported by the clinical data. According to RCTs, Freeze-all is indeed a beneficial strategy for hyper- responders or patients who follow PGT-A at blastocyst stage, but may not necessary improve the clinical, obstetric or perinatal outcomes of the treatment. In contradiction, it may lead to increased treatment cost, lab workload, embryo manipulation and time to live birth (44). For this reason, the policy should be carefully and individually applied to each patient.

In conclusion, in order to answer the question which of the patients that follow Freeze-all policy, may benefit more in terms of cumulative live birth rates, our results suggest that the number of formed and vitrified blastocysts, can be an essential criterion to evaluate the eventual treatment outcome. The present study tried to analyze the eventual outcome of the treatment, irrespectively to the number of the produced oocytes, but based on a more solid evidence, as the number of the available blastocysts. However, more in-depth analysis is required in order to identify the subgroups of patients who really are meant for cryopreservation and those who are meant for fresh embryo transfers. 


\section{DATA AVAILABILITY}

All datasets generated for this study are included in the manuscript and/or the supplementary files.

\section{REFERENCES}

1. Loutradi KE, Kolibianakis EM, Venetis CA, Papanikolaou EG, Pados G, Bontis I, et al. Cryopreservation of human embryos by vitrification or slow freezing: a systematic review and meta-analysis. Fertil Steril. (2008) 90:18693. doi: 10.1016/j.fertnstert.2007.06.010

2. Cobo A, de los Santos MJ, Castellò D, Gámiz P, Campos P, Remohí J. Outcomes of vitrified early cleavage-stage and blastocyst-stage embryos in a cryopreservation program: evaluation of 3,150 warming cycles. Fertil Steril. (2012) 98:1138-46.e1. doi: 10.1016/j.fertnstert.2012.07.1107

3. Rienzi L, Gracia C, Maggiulli R, LaBarbera AR, Kaser DJ, Ubaldi FM, et al. Oocyte, embryo and blastocyst cryopreservation in ART: systematic review and meta-analysis comparing slow-freezing versus vitrification to produce evidence for the development of global guidance. Hum Reprod Update. (2017) 23:139-55. doi: 10.1093/humupd/dmw038

4. Itskovitz-Eldor J, Kol S, Mannaerts B. Use of a single bolus of GnRH agonist triptorelin to trigger ovulation after $\mathrm{GnRH}$ antagonist ganirelix treatment in women undergoing ovarian stimulation for assisted reproduction, with special reference to the prevention of ovarian hyperstimulation syndrome: preliminary report: short communication. Hum Reprod. (2000) 15:19658. doi: 10.1093/humrep/15.9.1965

5. De Jong D, Van Hooren EG, Macklon NS, Mannaerts BM, Fauser BC. Pregnancy and birth after GnRH agonist treatment for induction of final oocyte maturation in a woman undergoing ovarian stimulation for ICSI, using a GnRH antagonist (Orgalutran/Antagon) to prevent a premature LH surge: a case report. J Assist Reprod Genet. (2001) 18:30-3. doi: 10.1023/a:1026498629324

6. Humaidan P, Bredkjaer HE, Bungum L, Grondahl ML, Westergaard L, Anderson CY. GnRH agonist (buserelin) or hCG for ovulation induction in GnRH antagonist IVF/ICSI cycles: a prospective randomized study. Hum Reprod. (2005) 20:1213-20. doi: 10.1093/humrep/deh765

7. Kolibianakis EM, Schultze-Mosgau A, Schroer A, van Steirteghem A, Devroey $\mathrm{P}$, Diedrich $\mathrm{K}$, et al. A lower ongoing pregnancy rate can be expected when GnRH agonist is used for triggering final oocyte maturation instead of HCG in patients undergoing IVF with GnRH antagonists. Hum Reprod. (2005) 20:2887-92. doi: 10.1093/humrep/dei150

8. Sunkara SK, Rittenberg V, Raine- Fenning N, Bhattacharya S, Zamora J, Coomarasamy A. Association between the number of eggs and live birth in IVF treatment: an analysis of 400135 treatment cycles. Hum Reprod. (2011) 26:1768-74. doi: 10.1093/humrep/der106

9. Drakopoulos P, Blockeel C, Stoop D, Camus M, de Vos M, Tournaye H, et al. Conventional ovarian stimulation and single embryo transfer for IVF/ICSI. How many oocytes do we need to maximize cumulative live birth rates after utilization of all fresh and frozen embryos? Hum Reprod. (2016) 31:3706. doi: 10.1093/humrep/dev316

10. Roque M, Lattes K, Serra S, Solà I, Geber S, Carreras R, et al. Fresh embryo transfer versus frozen embryo transfer in in vitro fertilization cycles: a systematic and meta-analysis. Fertil Steril. (2013) 99:15662. doi: 10.1016/j.fertnstert.2012.09.003

11. Cha J, Sun X, Dey SK. Mechanisms of implantation: strategies for successful pregnancy. Nat Med. (2012) 18:1754-67. doi: 10.1038/nm.3012

12. Shapiro BS, Daneshmand ST, Garner FC, Aguirre M, Hudson C, Thomas S. Evidence of impaired endometrial receptivity after ovarian stimulation for in vitro fertilization: a prospective randomized trial comparing fresh and frozen-thawed embryo transfer in high responders. Fertil Steril. (2011) 96:516-8. doi: 10.1016/j.fertnstert.2011. 02.059

13. Simon C, Velasco JJG, Valbuena D, Peinado JA, Moreno C, Remohí $\mathrm{J}$, et al. Increasing uterine receptivity by decreasing estradiol levels during the preimplantation period in high responders with the use of

\section{AUTHOR CONTRIBUTIONS}

All authors listed have made a substantial, direct and intellectual contribution to the work, and approved it for publication.

a follicle-stimulating hormone step-down regimen. Fertil Steril. (1998) 70:234-9. doi: 10.1016/S0015-0282(98)00140-X

14. Nikas G, Develioglu OH, Toner JP, Jones HW Jr. Endometrial pinopodes indicate a shift in the window of receptivity in IVF cycles. Hum Reprod. (1999) 14:787-92. doi: 10.1093/humrep/14.3.787

15. Richter KS, Shipley SK, McVearry I, Tucker MJ, Widra EA. Cryopreserved embryo transfers suggest that endometrial receptivity may contribute to reduced success rates of later developing embryos. Fertil Steril. (2006) 86:8626. doi: 10.1016/j.fertnstert.2006.02.114

16. Shapiro BS, Daneshmand ST, Garner FC, Aguirre M, Thomas S. Large blastocyst diameter, early blastulation, and low preovulatory serum progesterone are dominant predictors of clinical pregnancy in fresh autologous cycles. Fertil Steril. (2008) 90:302-9. doi: 10.1016/j.fertnstert.2007.06.062

17. Shapiro BS, Daneshmand ST, Garner FC, Aguirre M, Hudson C, Thomas S. Evidence of impaired endometrial receptivity after ovarian stimulation for in vitro fertilization: a prospective randomized trial comparing fresh and frozen-thawed embryo transfer in normal responders. Fertil Steril. (2011) 96:344-8. doi: 10.1016/j.fertnstert.2011.05.050

18. Griesinger G, von Otte S, Schroer A, Ludwig AK, Diedrich K, Al-Hasani S, et al. Elective cryopreservation of all pronuclear oocytes after $\mathrm{GnRH}$ agonist triggering of final oocyte maturation in patients at risk of developing OHSS: a prospective, observational proof-of-concept study. Hum Reprod. (2007) 22:1348-52. doi: 10.1093/humrep/dem006

19. Shapiro BS, Daneshmand ST, Garner FC, Aguirre M, Hudson C, Thomas S. High ongoing pregnancy rates after deferred transfer through bipronuclear oocyte cryopreservation and post-thaw extended culture. Fertil Steril. (2009) 92:1594-9. doi: 10.1016/j.fertnstert.2008.08.103

20. Manzanares MA, Gómez-Palomares JL, Ricciarelli E, Hernández ER. Triggering ovulation with gonadotropin-releasing hormone agonist in in vitro fertilization patients with polycystic ovaries does not cause ovarian hyperstimulation syndrome despite very high estradiol levels. Fertil Steril. (2010) 93:1215-9. doi: 10.1016/j.fertnstert.2008.12.019

21. Alviggi C, Conforti A, Crbone IF, Borrelli R, De Placido G, Guerriero S. Influence of cryopreservation on perinatal outcome after blastocystvs cleavage-stage embryo transfer: systematic review and metaanalysis. Ultrasound Obstet Gynecol. (2018) 51:54-63. doi: 10.1002/ uog. 18942

22. Maheshwari A, Pandey S, Amalraj Raja E, Shetty A, Hamilton M, Bhattacharya S. Is frozen embryo transfer better for mothers and babies? Can cumulative meta-analysis provide a definitive answer? Hum Reprod Update. (2018) 24:3558. doi: 10.1093/humupd/dmx031

23. Steward RG, Lan L, Shah AA, Yeh JS, Price TM, Goldfarb JM, et al. Oocyte number as a predictor for ovarian hyperstimulation syndrome and live birth: an analysis of 256,381 in vitro fertilization cycles. Fertil Steril. (2014) 101:96773. doi: 10.1016/j.fertnstert.2013.12.026

24. Polyzos NP, Drakopoulos P, Parra J, Pellicer A, Santos-Ribeiro S, Tournaye $\mathrm{H}$, et al. Cumulative live birth rates according to the number of oocytes retrieved after the first ovarian stimulation for in vitro fertilization/intracytoplasmic sperm injection: a multicenter multinational analysis including 15,000 women. Fertil Steril. (2018) 110:661-70.e1. doi: 10.1016/j.fertnstert.2018.04.039

25. Acharya KS, Acharya CR, Bishop K, Harris B, Raburn D, Muasher SJ. Freezing of all embryos in in vitro fertilization is beneficial in high responders, but not intermediate and low responders: an analysis of 82,935 cycles from the Society for Assisted Reproductive Technology registry. Fertil Steril. (2018) 110:880-7. doi: 10.1016/j.fertnstert.2018.05.024

26. Chang JC, Chen MJ, Guu HF, Chen YF, Yi YC, Kung HF, et al. Does the "freeze-all" policy allow for a better outcome in assisted reproductive techniques than the use of fresh embryo transfers? A retrospective study 
on cumulative live birth rates. Taiwan J Obstet Gynecol. (2017) 56:77580. doi: 10.1016/j.tjog.2017.10.013

27. Berkkanoglu M, Coetzee K, Bulut H, Ozgur K. Optimal embryo transfer strategy in poor response may include freeze-all. J Assist Reprod Genet. (2017) 34: 79-87. doi: 10.1007/s10815-016-0825-y

28. Barnhart KT. Are we ready to eliminate the transfer of fresh embryos in in vitro fertilization? Fertil Steril. (2014) 102:1-2. doi: 10.1016/j.fertnstert.2014.05.024

29. Blockeel C, Drakopoulos P, Santos-Ribeiro S, Polyzos NP, Tournaye H. A fresh look at the freeze-all protocol: a SWOT analysis. Hum Reprod. (2016) 31:491-7. doi: 10.1093/humrep/dev339

30. Roque M, Valle M, Kostolias A, Sampaio M, Geber S. Freeze-all cycle in reproductive medicine: current perspectives. JBRA Assist Reprod. (2017) 21:49-53. doi: 10.5935/1518-0557.20170012

31. Roque M, Valle M, Sampaio M, Geber S. Does freeze-all policy affect IVF outcomes in poor responders? Ultrasound Obstet Gynecol. (2017) 52:5304. doi: 10.1002/uog. 19000

32. Scott L, Alvero RJ, Leondires MP, Miller B. The morphology of human pronuclear embryos is positively related to blastocyst development and implantation. Hum Reprod. (2000) 15:2394403. doi: 10.1093/humrep/15.11.2394

33. Gardner DK, Schoolcraft WB. "In vitro culture of human blastocysts". In: Jansen R and Mortimer D, editors. Towards Reproductive Certainty: Infertility and Genetics Beyond 1999: The Plenary Proceedings of the 11th World Congress on in vitro Fertilization and Human Reproductive Genetics. Pearl River: Parthenon Press (1999). p. 378-88.

34. Van Landuyt L, De Vos A, Joris H, Verheyen G, Devroey P, Van Steirteghem A. Blastocyst formation in in vitro fertilization versus intracytoplasmic sperm injection cycles: influence of the fertilization procedure. Fertil Steril. (2005) 83:1397-403. doi: 10.1016/j.fertnstert.2004.10.054

35. Roque M, Valle M, Guimarães F, Sampaio M, Geber S. Freeze-all policy: fresh vs. frozen-thawed embryo transfer. Fertil Steril. (2015) 103:11903. doi: 10.1016/j.fertnstert.2015.01.045

36. Papanikolaou EG, Timotheou E, Tatsi P, Janssens H, Grynberg M, Athanasiadis A, et al. COMFFETI, combined fresh and frozen embryo transfers per individual: a new index of quality control for the performance of embryologic labs in the emerging era of segmentation of cycle and freeze-all strategy. 2019. Int J Fertil Steril. (2019) 12:339-42. doi: 10.22074/ijfs.2019.5424

37. Devroey P, Polyzos NP, Blockeel C. An OHSS-Free Clinic by segmentation of IVF treatment. Hum Reprod. (2011) 26:2593-7. doi: 10.1093/humrep/ der251

38. Pinborg A, Loft A, Henningsen AK, Rasmussen S, Andersen AN. Infant outcome of 957 singletons born after frozen embryo replacement: the Danish National Cohort Study 1995-2006. Fertil Steril. (2010) 94:13207. doi: 10.1016/j.fertnstert.2009.05.091

39. Henningsen AK, Pinborg A, Lidegaard $\varnothing$, Vestergaard C, Forman JL, Andersen AN. Perinatal outcome of singleton siblings born after assisted reproductive technology and spontaneous conception: Danish national sibling-cohort study. Fertil Steril. (2011) 95:959-63. doi: 10.1016/j.fertnstert.2010.07.1075

40. Wong KM, van Wely M, Mol F, Repping S, Mastenbroek S. Fresh versus frozen embryotransfers in assisted reproduction. Cohrane Database Syst Rev. (2017) 3:CD011184. doi: 10.1002/14651858.CD011184.pub2

41. D'Angelo A. Ovarian hyperstimulation syndrome prevention strategies: cryopreservation of all embryos. Semin Reprod Med. (2010) 28:5138. doi: $10.1055 / \mathrm{s}-0030-1265679$

42. Atkinson P, Koch J, Ledger WL. GnRH agonist trigger and a freeze-all strategy to prevent ovarian hyperstimulation syndrome: a retrospective study of OHSS risk and pregnancy rates. Aust N Z J Obstet Gynaecol. (2014) 54:581-5. doi: 10.1111/ajo.12277
43. Griesinger G, Schultz L, Bauer T, Broessner A, Frambach T, Kissler S. Ovarian hyperstimulation syndrome prevention by gonadotropin releasing hormone agonist triggering of final oocyte maturation in a gonadotropinreleasing hormone antagonist protocol in combination with a "freeze-all" strategy: a prospective multicentric study. Fertil Steril. (2011) 95:202933. doi: 10.1016/j.fertnstert.2011.01.163

44. Roque M, Haahr T, Geber S, Esteves SC, Humaidan P. Fresh versus elective frozen embryo transfer in IVF/ICSI cycles: a systematic review and meta-analysis of reproductive outcomes. Hum Reprod Update. (2019)25: 214. doi: 10.1093/humupd/dmy033

45. van der Gaast MH, Eijkemans MJC, van der Net JB, de Boer EJ, Burger CW, van Leeuwen FE, et al. Optimum number of oocytes for a successful first IVF treatment cycle. Reprod BioMed Online. (2006) 13:47680. doi: 10.1016/S1472-6483(10)60633-5

46. Ji J, Liu Y, Tong XH, Luo L, Ma J, Chen Z. The optimum number of oocytes in IVF treatment: an analysis of 2455 cycles in China. Hum Reprod. (2013) 28:2728-34. doi: 10.1093/humrep/det303

47. Vaughan DA, Leung A, Resetkova N, Ruthazer R, Penzias AS, Sakkas D, et al. How many oocytes are optimal to achieve multiple live births with one stimulation cycle? The one-and-done approach. Fertil Steril. (2017) 107:397404.e3. doi: 10.1016/j.fertnstert.2016.10.037

48. Zhu Q, Chen Q, Wang L, Lu X, Lyu Q, Wang Y, et al. Live birth rates in the first complete IVF cycle among 20687 women using a freeze-all strategy. Hum Reprod. (2018) 33:924-9. doi: 10.1093/humrep/dey044

49. Smith A, Tilling K, Nelson SM, Lawlor DA. Live-birth rate associated with repeat in vitro fertilization treatment cycles. JAMA. (2015) 314:265462. doi: 10.1001/jama.2015.17296

50. McLernon DJ, Steyerberg EW, Te Velde ER, Lee AJ, Bhattacharya S. Predicting the chances of a live birth after one or more complete cycles of in vitro fertilisation: population based study of linked cycle data from 113873 women. BMJ. (2016) 355:i5735. doi: 10.1136/bmj.i5735

51. Rubio C, Mercader A, Alamá P, Lizán C, Rodrigo L, Labarta E, et al. Prospective cohort study in high responder oocyte donors using two hormonal stimulation protocols: impact on embryo aneuploidy and development. Hum Reprod. (2010) 25:2290-7. doi: 10.1093/ humrep/deq174

52. Labarta E, Bosch E, Mercader A, Alamá P, Mateu E, Pellicer A. A higher ovarian response after stimulation for IVF is related to a higher number of euploid embryos. Biomed Res Int. (2017) 2017:5637923. doi: 10.1155/2017/5637923

53. Ata B, Kaplan B, Danzer H, Glassner M, Opsahl M, Tan SL, et al. Array CGH analysis shows that aneuploidy is not related to the number of embryos generated. Reprod BioMed Online. (2012) 24:61420. doi: 10.1016/j.rbmo.2012.02.009

54. Munné S, Alikani M, Ribustello L, Colls P, Martínez-Ortiz PA, McCulloh DH. Euploidy rates in donor egg cycles significantly differ between fertility centers. Hum Reprod. (2017) 32:743-9. doi: 10.1093/humrep/dex031

Conflict of Interest Statement: The authors declare that the research was conducted in the absence of any commercial or financial relationships that could be construed as a potential conflict of interest.

Copyright () 2019 Papanikolaou, Chartomatsidou, Timotheou, Tatsi, Katsoula, Vlachou, Asouchidou, Zafeiratis and Najdecki. This is an open-access article distributed under the terms of the Creative Commons Attribution License (CC BY). The use, distribution or reproduction in other forums is permitted, provided the original author(s) and the copyright owner(s) are credited and that the original publication in this journal is cited, in accordance with accepted academic practice. No use, distribution or reproduction is permitted which does not comply with these terms. 